\title{
Pinning down response inhibition in the brain - Conjunction analyses of the Stop-signal task
}

\author{
Boehler C.N. ${ }^{\text {a,b,c,* }}$, Appelbaum L.G. ${ }^{\text {, }}$ Krebs R.M. ${ }^{\text {a }}$, Hopf J.M. ${ }^{\text {b,c }}$, Woldorff M.G. ${ }^{\text {a,d }}$ \\ a Center for Cognitive Neuroscience, Duke University, Durham, NC 27708, USA \\ b Leibniz-Institute for Neurobiology, Brenneckestr. 6, D-39118 Magdeburg, Germany \\ c Department of Neurology, Otto-von-Guericke-University, Leipziger Str. 44, D-39120 Magdeburg, Germany \\ d Department of Psychiatry, Duke University, Durham, NC 27708, USA
}

\section{A R T I C L E I N F O}

\section{Article history:}

Received 10 October 2009

Revised 20 March 2010

Accepted 30 April 2010

Available online $\mathrm{xxxx}$

\section{Keywords:}

Inhibitory motor control

Cognitive conjunction

Inferior frontal gyrus

Visual attention

Anterior insula

\begin{abstract}
A B S T R A C T
Successful behavior requires a finely-tuned interplay of initiating and inhibiting motor programs to react effectively to constantly changing environmental demands. One particularly useful paradigm for investigating inhibitory motor control is the Stop-signal task, where already-initiated responses to Gostimuli are to be inhibited upon the rapid subsequent presentation of a Stop-stimulus (yielding successful and unsuccessful Stop-trials). Despite the extensive use of this paradigm in functional neuroimaging, there is no consensus on which functional comparison to use to characterize response-inhibition-related brain activity. Here, we utilize conjunction analyses of successful and unsuccessful Stop-trials that are each contrasted against a reference condition. This conjunction approach identifies processes common to both Stop-trial types while excluding processes specific to either, thereby capitalizing on the presence of some response-inhibition-related activity in both conditions. Using this approach on fMRI data from human subjects, we identify a network of brain structures that was linked to both types of Stop-trials, including lateral-inferior frontal and medial frontal cortical areas and the caudate nucleus. In addition, comparisons with a reference condition matched for visual stimulation identified additional activity in the right inferior parietal cortex that may play a role in enhancing the processing of the Stop-stimuli. Finally, differences in stopping efficacy across subjects were associated with variations in activity in the left anterior insula. However, this region was also associated with general task accuracy (which furthermore correlated directly with stopping efficacy), suggesting that it might actually reflect a more general mechanism of performance control that supports response inhibition in a relatively nonspecific way.
\end{abstract}

(c) 2010 Elsevier Inc. All rights reserved.

\section{Introduction}

Successful behavior requires continuous coordination between the initiation and inhibition of actions, the latter being particularly important when sudden changes in the situation call for an alteration, or even the cancellation, of a planned or ongoing behavior. One of the most prominent experimental paradigms designed to investigate response inhibition is the Stop-signal task (Logan, 1994; Logan et al., 1984). In this task, a Go-stimulus requiring a choice-reaction is rapidly followed, on some trials, by a Stop-stimulus that requires the subjects to withhold the response they were about to give.

Computational models have captured the dynamics of the underlying neural processes as a 'horse race' between Go- and Stopprocesses that are believed to evolve mostly independently over time (Boucher et al., 2007; de Jong et al., 1990; Logan et al., 1984;

\footnotetext{
* Corresponding author. Center for Cognitive Neuroscience, Duke University, B203 Levine Science Research Center, Box 90999, Durham, NC 27708, USA. Fax: +1 919 6810815.

E-mail address: nico.boehler@duke.edu (C.N. Boehler).
}

Verbruggen and Logan, 2008). Depending on whether the Stopprocess can be implemented sufficiently before the Go-process reaches a certain threshold, inhibition will either succeed, or not. One important contribution of this race model is that it provides a formal means to infer the time required for the Stop-process to catch up to the Go-process, called the Stop-signal response time (SSRT). Based on this analysis, subjects typically need around $200 \mathrm{~ms}$ to stop an already-initiated behavioral response (Logan and Cowan, 1984; Logan et al., 1984). Moreover, it can be derived from the model that some response inhibition should also be entailed in unsuccessful Stop-trials. Although in such trials the Stop-process loses the race against the Go-process, it will nevertheless usually be initiated and have proceeded partway.

Recent years have seen enormous interest in the Stop-signal task. This popularity speaks to the central role that inhibitory control appears to play in normal human behavior, normal cognitive development, and in a range of neurological and psychiatric conditions such as attention-deficit hyperactivity disorder (ADHD) and substance abuse (Aron, 2009; Aron et al., 2007b; Groman et al., 2009; Verbruggen and Logan, 2008; Williams et al., 1999), as well as 
to the merits of this paradigm for investigating inhibitory control processes. The neural mechanisms underlying response inhibition have been investigated employing a wealth of approaches, including neuropsychological, TMS, EMG, EEG, MEG, and fMRI studies in humans, as well as neurophysiological recordings in animals. Converging evidence from these different methodologies has led to the view that a predominantly right-hemisphere network of mainly three brain areas is crucial for response inhibition: inferior frontal gyrus (IFG; especially the frontal operculum extending into the insula), medial frontal areas (particularly the pre-supplementary motor area; pre-SMA), and the basal ganglia (for recent reviews see Aron et al., 2007b; Chambers et al., 2009; Verbruggen and Logan, 2008). The dominant view contends that, in response to a Stopstimulus, a signal from the inferior and/or medial frontal cortex is sent to the basal ganglia to cancel the motor program triggered by the Gostimulus. This input has been proposed to enter the basal ganglia either through a so-called "hyperdirect" route to the subthalamic nucleus (STN), or the "indirect" route via the striatum (consisting of the caudate nucleus and the putamen; Aron et al., 2007a; Aron and Poldrack, 2006; Eagle et al., 2008; Mink, 1996; Nambu et al., 2002; Ray et al., 2009). Interactions between the different parts of the basal ganglia and the associated STN then give rise to a signal that is sent via the thalamus to motor cortex, where the response is ultimately inhibited (Stinear et al., 2009).

While neuroimaging studies have substantially contributed to our knowledge of the neural mechanisms that support response inhibition, a consensus has not been reached as to the appropriate functional comparison that should be used to isolate processes related to response inhibition. Consequently, approaches and results differ quite substantially between studies (Verbruggen and Logan, 2008). When devising a functional contrast for the Stop-signal task, a couple of factors have to be considered (see supplementary Table S1): response inhibition is almost certainly present in both successful and unsuccessful Stop-trials ${ }^{1}$ (Garavan et al., 2002), although it seems likely to be more pronounced, or at least faster, for successful Stoptrials. Conversely, there are also operations likely to be specific for either type of Stop-trial, such as registering the positive outcome of a successful Stop-trial ${ }^{2}$ or evaluating the error in an unsuccessful one, which are not directly related to response inhibition. Finally, both types of Stop-trials differ greatly from Go-trials in such important characteristics as their sensory stimulation and the frequency with which they occur.

Two main approaches have been commonly used to identify inhibition-related brain activity in neuroimaging studies of the Stopsignal task. In one approach, activity is isolated by contrasting successful and unsuccessful Stop-trials (e.g., Duann et al., 2009; Li et al., 2006; Rubia et al., 2003; Rubia et al., 2007). In the other, Stop-trials (either all Stop-trials or successful Stop-trials alone) are compared to Go-trials (e.g., Aron and Poldrack, 2006; Pliszka et al., 2006; Ramautar et al., 2006; Rubia et al., 2001). While both approaches have advantages for extracting differential activity of interest, each one has some distinct limitations. Of concern in the first approach (comparing successful vs. unsuccessful Stop-trials) is the underlying assumption that inhibitory processes are considerably more pronounced in successful than in unsuccessful Stop-trials. Therefore, to the degree that response-inhibition-related activity is also present in unsuccessful Stop-trials, this approach is overly conservative. Additionally, successful Stop-trials might entail specific neural operations that are not directly related to response inhibition, such as registering the successful outcome of Stop-trials. Such processes might be

\footnotetext{
1 This is particularly likely in paradigms that constantly track behavior and keep it in a range where subjects just barely succeed or fail to inhibit their response.

2 Such outcome evaluation, in turn, is probably different from registering the successful outcome of Go-trials, since Go-trials are more prevalent and are virtually always responded to correctly.
}

mistaken as being directly related to response inhibition, as the employed contrast cannot differentiate between them.

The second approach (comparing Stop-trials and Go-trials) is also limited in that some processes are likely to be present in Stop-trials and absent in Go-trials that are also not directly related to response inhibition (see supplemental Table S1). Most prominently, there are differences in visual stimulation in these two conditions (i.e., there is no Stop-stimulus in Go-trials), which thereby render interpretations of visually responsive areas rather problematic. Importantly, some of these areas might actually play an integral role in response inhibition and deserve investigation. A good example is the parietal cortex, where large swaths of activity have been very often associated with motor control tasks (e.g., Aron and Poldrack, 2006; Coxon et al., 2009; Garavan et al., 2002; Jaffard et al., 2008; Menon et al., 2001). Despite being identified in motor control experiments so regularly, it is not clear whether some parietal regions indeed subserve a function that is directly related to response inhibition (Bunge et al., 2002; Chambers et al., 2009; Coxon et al., 2009; Wager et al., 2005), or whether its functions might be restricted to the attentive processing of the relevant stimuli. In order to make distinctions between such processes, it is crucial to separate out effects arising from the inherent differences in sensory stimulation between Stop- and Go-trials.

To summarize, it appears that both approaches that have been commonly used thus far have some limitations. Comparing successful and unsuccessful Stop-trials is potentially overly conservative, whereas comparing Stop-trials and Go-trials has limited specificity. The present study attempts to find a compromise between these two extremes by utilizing conjunction analyses of fMRI responses to isolate cognitive/neural operations that are shared between successful and unsuccessful Stop-trials, as compared to trials in which there is no response inhibition. This strategy should account for specificity issues arising from processes that are exclusive to either Stop-trial type, in that it excludes activity that is only present in one condition. However, as detailed above, there are also factors that are common to both Stop-trial types beyond those directly related to response inhibition. As noted above, the most prominent of these factors is the processing of the Stop-stimulus that makes activity differences between Stop-trials and Go-trials in sensory areas hard to interpret. Accordingly, in order to allow investigation of hemodynamic responses in areas that respond to sensory stimulation, the present study included a sensory control condition that was matched in visual stimulation to the Stop-trials but did not require response inhibition. This approach should thus be more specific than the comparison between Stop-trials and Go-trials, while at the same time it is likely to be less restrictive than the comparison of successful and unsuccessful Stop-trials, depending on how much more pronounced responseinhibition activity is in successful than in unsuccessful Stop-trials.

\section{Materials and methods}

\section{Participants}

Eighteen subjects participated in this study, two of which were excluded due to technical problems. One additional subject was excluded due to particularly poor behavioral performance. Of the 15 remaining participants, nine were female and the mean age was 22.9 years. All subjects had correct or corrected-to-normal visual acuity, and none of them reported a history of psychiatric or neurological disorders. All were paid for participation and gave written informed consent before the experiment in accordance with the Duke Institutional Review Board.

\section{Task}

There were two main tasks in the experiment, performed in different blocks that alternated once during each run: a classical Stop-signal task 
in which the Stop signals were relevant (Stop-relevant (SR) blocks), in which subjects were to try to withhold their response following the Stop-stimulus, and a second task with identical visual stimulation, but with instructions to ignore the presentation of the Stop-signs and to thus not withhold the response (Stop-irrelevant (SI) blocks). For clarity, we append the suffixes "_SR" and "_SI" to the abbreviations of the conditions to indicate that the Stop-signs were relevant or irrelevant in a given block. Each task was performed once per experimental run, and the two task blocks were separated by a 16-s break (i.e., task break, while the MR scanner was not stopped). Odd runs began with the SR task, followed by the SI task within the same run, with even runs in the opposite order. A total of 10 runs were collected, yielding a total of approximately 950 trials. The time between trial onsets was varied pseudo-randomly between 2 and $8 \mathrm{~s}$ yielding a gamma distribution (average $3.2 \mathrm{~s}$ ) to allow for the separation of different conditions in an event-related fMRI analysis (Hinrichs et al., 2000).

\section{Stop-relevant (SR) blocks}

These blocks used a standard Stop-signal task (using German traffic-light signs, see Fig. 1A), with two types of trials presented in a random sequence: frequent Go-trials (GTs), where a rapid response to a choice-reaction stimulus was required, and less-frequent Stop-trials (STs), where the presentation of a Stop-sign briefly following the choice-reaction stimulus indicated that the response was to be stopped. In the present experiment, Go-trials accounted for $80 \%$ of all trials. In these, a green symbol was presented for $800 \mathrm{~ms}$, and subjects had to decide whether the profile of a walking figure was oriented to the left or right (mapped to the right index and middle finger). Stop-trials (20\% of trials) started identically to Go-trials, but after a variable stimulus onset asynchrony (SOA) the Go-stimulus was replaced by a red Stop-sign until the end of the total stimulus duration, signaling subjects to withhold their response. Total stimulus duration was kept constant at $800 \mathrm{~ms}$.

The SOA between the Go- and the Stop-stimulus is an important factor determining whether subjects accomplish withholding the motor response (successful Stop-trials, SST) or fail to inhibit their response (called unsuccessful Stop-trials, UST; see Logan, 1994). Therefore, the Go-Stop SOA is usually titrated using an adaptive staircase procedure so as to yield an approximately equivalent number of SSTs and USTs. In the present experiment we followed this approach, increasing the SOA by $17 \mathrm{~ms}$ (one refresh screen) after a SST and decreasing it by the same amount after an UST. The initial SOA was $200 \mathrm{~ms}$. This procedure furthermore allowed us to calculate the Stopsignal response time (SSRT) by subtracting the mean Go-Stop SOA from the average Go-trial response time (Logan, 1994), an approach that is generally viewed as providing a reliable estimate for the SSRT in situations where the proportion of successful and unsuccessful Stoptrials is about even (Verbruggen and Logan, 2009). Descriptively, this SOA characterizes the time at which the Stop-process triggered by the Stop-stimulus will manage in half of the trials to catch up with the Goprocess soon enough to cancel the response.

\section{Stop-irrelevant (SI) blocks}

The Stop-irrelevant blocks were composed of the same visual stimulation as in the Stop-relevant blocks (Fig. 1B). The crucial difference was that in the SI-blocks subjects were instructed to respond to all Go-stimuli irrespective of whether or not they were followed by a Stop-stimulus. In an attempt to equate the sensory stimulation as much as possible to that in the SR-blocks, we also varied the SOA between the Go- and the Stop-stimulus in a similar manner. In particular, the SOA resulting from the staircase procedure of the preceding SR-block was used as the initial value, which was then varied in a random one-up/ one-down fashion after each "Stop"-trial within plus/minus three 17-ms steps of the initial value. The staircase for the next SR-block was then taken from the end of the preceding SR-block (i.e., ignoring the random variation that happened during the SI-block).

\section{Data acquisition and basic analysis}

MR data was acquired on a 3-Tesla GE Signa MRI system. Highresolution structural T1 (3D Fast Spoiled Gradient Recalled (FSPGR); $1 \times 1 \times 1 \mathrm{~mm}$ resolution) and proton density (PD)/T2 weighted images (2-D Fast Spin Echo (FSE); $1 \times 1 \times 1 \mathrm{~mm}$ resolution) were acquired for each subject. Functional images were acquired with a reverse spiral imaging sequence $\left(\mathrm{TR}=2000 \mathrm{~ms}, \mathrm{TE}=25 \mathrm{~ms}\right.$; flip angle $=75^{\circ} ; 32$ slices with $3 \times 3 \times 3 \mathrm{~mm}$ resolution; AC-PC orientation providing coverage approximately from the top of the brain down to the Pons). The first five functional images were discarded from the analysis, to allow for reaching steady-state magnetization.

The fMRI data were pre-processed and statistically analyzed using the SPM5 software package (Wellcome Department of Imaging Neuroscience, Institute of Neurology, London, UK). All functional images were slice-time corrected, spatially aligned, and normalized applying the normalization parameters used to warp the highresolution T1 image to the SPM template. After resampling to a resolution of $2 \times 2 \times 2 \mathrm{~mm}$, the functional images were smoothed with an isotropic 8 -mm full-width half-maximum Gaussian kernel and highpass-filtered (cut-off $128 \mathrm{~s}$ ). For each subject, a statistical model was computed by applying a canonical hemodynamic response function (HRF) combined with time and dispersion derivatives for each of the conditions (Friston et al., 1998). All conditions were modeled separately, and only the trials with correct responses were

\section{A Stop-relevant blocks (SR)}

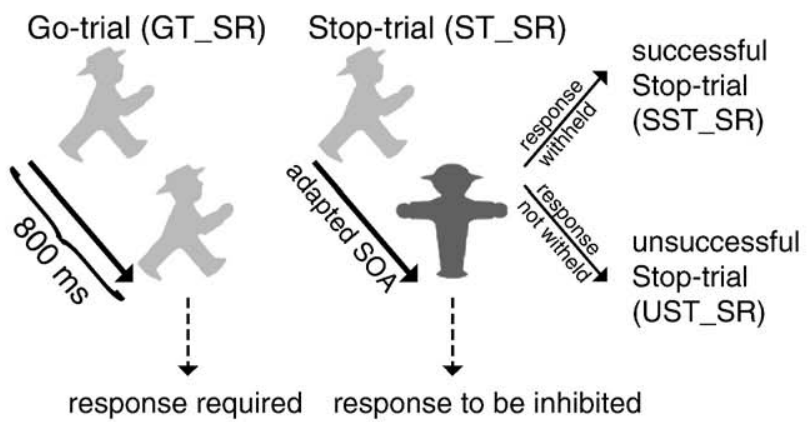

\section{B Stop-irrelevant blocks (SI)}

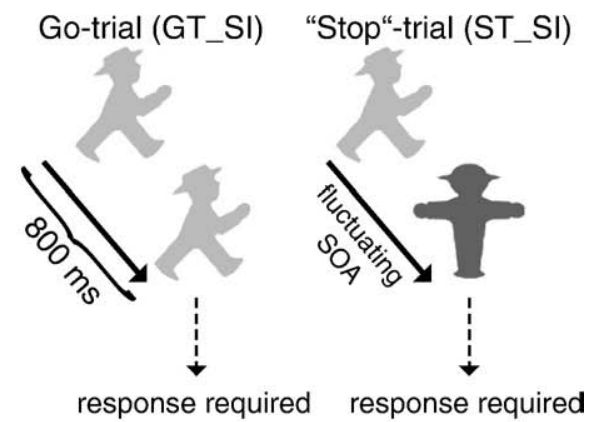

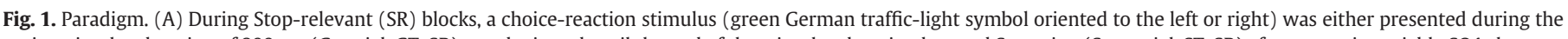

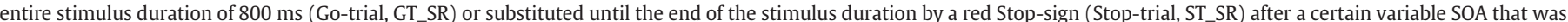

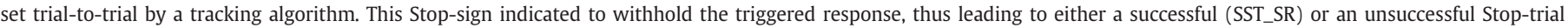

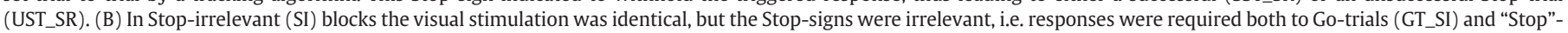
trials (ST_SI). 
included (or with no response in the case of successful Stop-trials). Additional regressors were included to model all events of no interest (trials with incorrect responses, misses, and the onset of the break), as well as the six covariates per session containing the realignment parameters capturing the subjects' movements during the experiment.

The parameter estimates resulting from each condition/contrast and subject (first-level analysis) were entered into a second-level, randomeffects group analysis using one-sample $t$-tests that were thresholded at $p<0.001$ (uncorrected) and $k=10$ contiguous voxels for simple contrasts. Additionally, a more liberal threshold was employed for subcortical areas ( $p<0.005$ and $k=5$ contiguous voxels). Submaxima within a contiguous cluster in the cortex are reported if they are more than $12 \mathrm{~mm}$ apart. In general, we opted for these relatively liberal (uncorrected) threshold levels for two reasons: first, our key results were predicted a priori. We did not aim to identify areas that have never before been reported in the context of response inhibition, but rather evaluate the presence of well-known structures in different functional contrasts. Therefore, multiple-comparison correction with respect to the entire search volume would be overly conservative. The more liberal thresholds for subcortical areas, where search volumes for predicted areas would be smaller than for most cortical areas, were chosen in an attempt to replicate previous reports of basal ganglia and STN involvement in response inhibition (e.g., Aron and Poldrack, 2006; Li et al., 2008; Rubia et al., 2007; Vink et al., 2005). As a consequence of this approach, we focus in our analysis and discussion on those areas that have been identified by previous imaging studies that have utilized variants of the Stop-signal task. Conversely, areas arising somewhat unexpectedly should be interpreted with care since they could represent type-I errors, and are indicated as such when referred to. Secondly, a more liberal threshold is generally more appropriate for statements concerning the absence of activity in certain areas. Small structures - e.g., in the basal ganglia - are particularly unlikely to display activity differences that are pronounced enough to still be identified by more conservative approaches.

For visualization purposes, activation maps were overlaid on the grand-average normalized $\mathrm{T} 1$ anatomical image across subjects using MRIcro (http://www.sph.sc.edu/comd/rorden/mricro.html). Statistical assessment of the behavioral data was accomplished by means of repeated-measures analyses of variance (rANVOA), with a nonsphericity correction of the degrees of freedom (Greenhouse-Geisser algorithm) where necessary.

\section{Conjunction analyses}

The current experiment was designed to identify brain areas that subserve functions related to inhibitory motor control that are shared by both successful and unsuccessful Stop-trials. To this end, three types of conjunction analyses were employed that each combined the results of separate one-sample $t$-tests ( see also Supplementary Table S1): (1) We performed a conjunction of the separate contrasts for successful and unsuccessful Stop-trials versus Go-trials from the Stop-relevant (SR) blocks ('SST_SR > GT_SR' and 'UST_SR>GT_SR'; “within-SR-block conjunction"). (2) We performed a conjunction of the separate contrasts for successful and unsuccessful Stop-trials from the Stop-relevant (SR) blocks versus the "Stop"-trials from the Stop-irrelevant (SI) blocks ('SST_SR > ST_SI' and 'UST_SR>ST_SI'; "between-block conjunction"). (3) We then combined these two conjunctions by performing a secondlevel conjunction between them ("second-level conjunction").

All three of these conjunctions were formulated as conjunction null hypotheses (Friston et al., 2005; Nichols et al., 2005) and should therefore only yield activations that are significantly present in both original contrasts of the conjunction (also referred to as minimum statistic). That is, conjunctions represent a logical "and", requiring both contrasts to be separately significant for the conjunction to be significant. As this type of conjunction analysis is relatively susceptible to slight spatial variations of activity in the two simple contrasts, we opted for slightly more liberal significance thresholds $(p<0.002$, $k=10$ and $p<0.01, k=5$ for subcortical areas). Again, this significance level is only appropriate for analyses directed toward results predicted a priori based on the existing literature. In an additional ROI analysis, parameter estimates were extracted from the local maxima of the between-block conjunction contrast using the MarsBar software package (http://marsbar.sourceforge.net/).

\section{Brain-behavior correlations}

In a set of additional analyses, we correlated fMRI activity in different brain areas with behavioral measures (mainly the SSRT) across subjects. In order to tap into potential differences between different submaxima within larger clusters, and to account for the relatively small cluster sizes in some regions, we extracted activity estimates from the peak voxels within the local maxima identified by the between-block conjunction (i.e., the conjunction of the successful and unsuccessful Stop-trials relative to the Stop-irrelevant "Stop"-trials as a reference). Specifically, we extracted the differences of the beta values corresponding to the contrasts "SST_SR $>$ ST_SI" and "UST_SR $>$ ST_SI", and averaged the two resulting values for each subject. Thus, these values represent the degree to which brain areas were more active during Stop-relevant Stop-trials than during Stop-irrelevant ones. These values were then correlated across subjects with the behavioral parameters.

\section{Results}

\section{Behavioral results}

An overview of the main behavioral parameters is provided in Table 1 . Subjects performed very accurately under both task regimes, with similar accuracy in the three conditions that require responses (i.e., Go-trials in both the SR and SI task blocks and "Stop"-trials in SIblocks; $F(1.5,21.4)=2.2, p=0.15$ ). Response times (RTs) were the slowest on Stop-relevant Go-trials, as compared to unsuccessful Stop-trials from the same blocks and Go- and Stop-trials from the Stop-irrelevant blocks (overall $F$-test: $F(1.8,25.3)=32.3, p<0.001$ ), but were similar between these other three conditions $(F(1.3,17.61)=0.9$, $p=0.37)$. During SR-block Stop-trials, subjects managed to withhold their behavioral response on approximately half of the trials, indicating the effectiveness of the staircase SOA-adjustment procedure we used that was aimed at achieving approximately equal trial numbers for the two conditions. The average SSRT was $230 \mathrm{~ms}$, which is within the typical range.

\section{Functional comparison of successful and unsuccessful Stop-trials (SR-blocks)}

Consistent with our notion that this contrast might be overly conservative in characterizing response inhibition, we only found a few, relatively weakly activated areas that were more active for successful than unsuccessful Stop-trials (see Table 2 and Fig. 2).

Table 1

Behavioral data (average $+/-\mathrm{SD}$ ).

\begin{tabular}{lc}
\hline Stop-relevant (SR) blocks & \\
\hline Correct Go-trials & $97.6 \%(+/-2.1)$ \\
Proportion SST & $52.7 \%(+/-3.8)$ \\
RT Go-trials & $520 \mathrm{~ms}(+/-84)$ \\
RT unsuccessful Stop-trials & $446 \mathrm{~ms}(+/-64)$ \\
SSRT & $230 \mathrm{~ms}(+/-26)$ \\
& \\
Stop-irrelevant (SI) blocks & \\
\hline Correct Go-trials & $96.6 \%(+/-2.5)$ \\
Correct “Stop"-trials & $97.1 \%(+/-3)$ \\
RT Go-trials & $436 \mathrm{~ms}(+/-48)$ \\
RT Stop-trials & $439 \mathrm{~ms}(+/-44)$ \\
\hline
\end{tabular}


Table 2

fMRI activations for the contrast SST_SR > UST_SR.

\begin{tabular}{|c|c|c|c|c|c|c|}
\hline \multirow[t]{2}{*}{ Anatomical structure } & \multirow[t]{2}{*}{ Hemisphere } & \multirow[t]{2}{*}{$\begin{array}{l}\text { Cluster size } \\
\text { [voxel] }\end{array}$} & \multirow[t]{2}{*}{$t$-value } & \multicolumn{3}{|c|}{$\begin{array}{l}\text { Peak coordinates } \\
\text { MNI (mm) }\end{array}$} \\
\hline & & & & $x$ & $y$ & $z$ \\
\hline \multicolumn{7}{|l|}{ Frontal cortex } \\
\hline Inferior frontal gyrus & $\mathrm{R}$ & 26 & 4.37 & 50 & 10 & 24 \\
\hline \multicolumn{7}{|l|}{ Occipital cortex } \\
\hline Superior occipital gyrus & $\mathrm{R}$ & 33 & 4.95 & 18 & -96 & 16 \\
\hline \multicolumn{7}{|l|}{ Subcortical areas ${ }^{\mathrm{a}}$} \\
\hline Caudate nucleus & $\mathrm{L}$ & 9 & 3.45 & -14 & 22 & 4 \\
\hline
\end{tabular}

Data are thresholded at $p<0.001$ (uncorrected), with a cluster-level of $k=10$.

a More liberal threshold for subcortical areas ( $p<0.005$ (uncorrected), $k=5$ ).

Specifically, these areas were found in the right occipital cortex, inferior frontal gyrus (IFG), and the left caudate nucleus. Activity in the IFG and the caudate nucleus might indeed reflect stronger engagement of processes related to response inhibition. These two activation differences were not very prominent, however, and they seem unlikely to accurately reflect the full set of activations underlying inhibitory control processes. Thus, various areas usually implicated in this process were only modestly active (such as the caudate nucleus) or were entirely absent (e.g., the right frontal operculum that is inferior to the IFG spot that the present analysis identified), supporting the view that the inhibitory control was exerted to a relatively similar degree in successful and unsuccessful Stop-trials.

\section{Functional comparison of successful Stop-trials and Go-trials (SR-blocks)}

Consistent with the difference in stimulation, the contrast between successful Stop-trials and the Go-trials not only yielded numerous differences in a wide network of frontal control regions, but also identified a large set of effects in temporal and occipital areas (Table 3). Many if not most of these latter areas are presumably

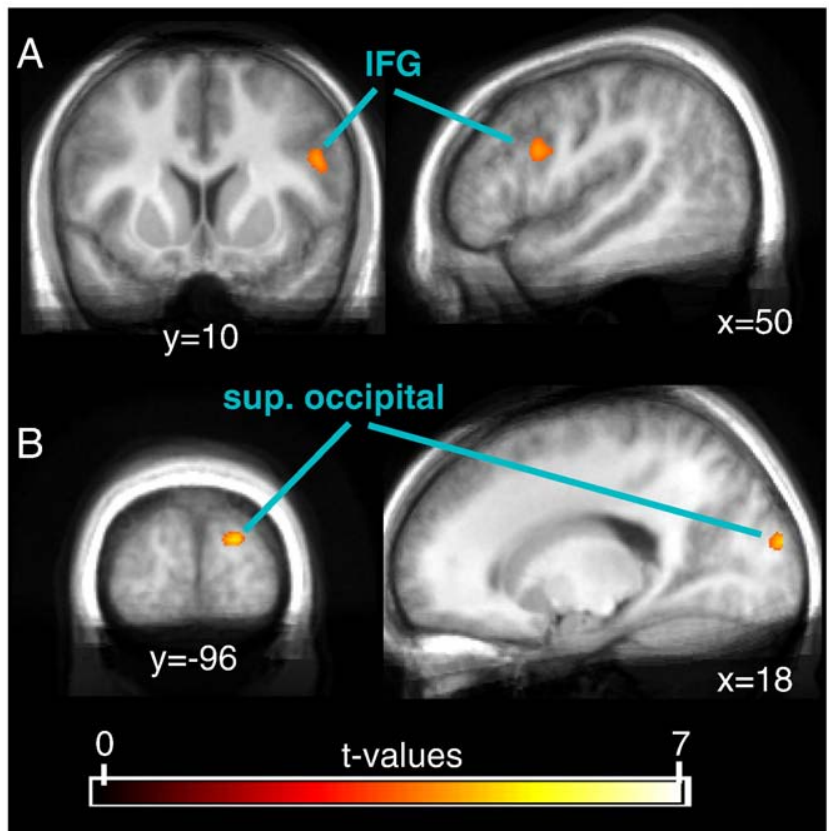

Fig. 2. Results of the direct comparison between successful and unsuccessful Stop-trials (SST_SR > UST_SR). This contrast revealed cortical activity in the right IFG (A) and the right superior occipital cortex (B), and subcortical activity in the left caudate nucleus (not shown in figure). The displayed data was thresholded at a $t$-value of 3.5.
Table 3

fMRI activations for the contrast SST_SR $>$ GT_SR

\begin{tabular}{|c|c|c|c|c|c|c|}
\hline \multirow[t]{2}{*}{ Anatomical structure } & \multirow[t]{2}{*}{ Hemisphere } & \multirow[t]{2}{*}{$\begin{array}{l}\text { Cluster size } \\
\text { [voxel] }\end{array}$} & \multirow[t]{2}{*}{$t$-value } & \multicolumn{3}{|c|}{$\begin{array}{l}\text { Peak coordinates } \\
\text { MNI (mm) }\end{array}$} \\
\hline & & & & $x$ & $y$ & $z$ \\
\hline \multicolumn{7}{|l|}{ Frontal cortex } \\
\hline Insula & $\mathrm{R}$ & 587 & 12.05 & 38 & 14 & -2 \\
\hline Frontal operculum & & & 5.19 & 42 & 30 & 2 \\
\hline Inferior frontal gyrus & $\mathrm{R}$ & 377 & 9.32 & 48 & 14 & 32 \\
\hline Middle frontal gyrus & & & 4.89 & 36 & 2 & 56 \\
\hline Middle frontal gyrus & & & 4.84 & 48 & 6 & 48 \\
\hline Frontal operculum/insula & $\mathrm{L}$ & 460 & 6.36 & -44 & 22 & 6 \\
\hline Inferior frontal gyrus & & & 5.35 & -38 & 22 & 20 \\
\hline Middle frontal gyrus & $\mathrm{R}$ & 65 & 4.86 & 38 & 38 & 30 \\
\hline Middle frontal gyrus & $\mathrm{L}$ & 15 & 4.84 & -28 & 2 & 58 \\
\hline Pre-SMA & $\mathrm{L} / \mathrm{R}$ & 76 & 4.8 & 0 & 20 & 48 \\
\hline Pre-SMA & & & 3.94 & 12 & 18 & 50 \\
\hline Middle frontal gyrus & $\mathrm{L}$ & 70 & 4.63 & -34 & 38 & 34 \\
\hline ACC & $\mathrm{R}$ & 18 & 4.53 & 6 & 26 & 22 \\
\hline Pre-central gyrus & $\mathrm{L}$ & 11 & 4.27 & -50 & 4 & 42 \\
\hline Pre-central gyrus & $\mathrm{L}$ & 15 & 4.18 & -40 & 2 & 36 \\
\hline \multicolumn{7}{|l|}{ Parietal and temporal cortex } \\
\hline Middle temporal gyrus & $\mathrm{R}$ & 4086 & 9.68 & 40 & -60 & 16 \\
\hline Inferior parietal lobule & & & 7.81 & 60 & -42 & 26 \\
\hline Inferior parietal lobule & & & 7.51 & 50 & -36 & 44 \\
\hline Middle temporal gyrus & $\mathrm{R}$ & 132 & 6.51 & 48 & -26 & -6 \\
\hline Inferior parietal lobule & $\mathrm{L}$ & 1030 & 5.54 & -52 & -38 & 50 \\
\hline Inferior parietal lobule & & & 5.48 & -62 & -40 & 28 \\
\hline \multicolumn{7}{|l|}{ Temporal and occipital cortex } \\
\hline Middle occipital gyrus & $\mathrm{L}$ & 805 & 8.14 & -44 & -86 & 4 \\
\hline Inferior occipital gyrus & & & 5.97 & -40 & -62 & -8 \\
\hline Middle temporal gyrus & & & 4.93 & -60 & -66 & 10 \\
\hline Middle occipital gyrus & $\mathrm{L}$ & 21 & 5.56 & -28 & -74 & 20 \\
\hline \multicolumn{7}{|l|}{ Subcortical areas ${ }^{\mathrm{a}}$} \\
\hline Thalamus & $\mathrm{L} / \mathrm{R}$ & 308 & 5.43 & 4 & -16 & -2 \\
\hline Superior colliculus & & & 4.89 & 0 & -28 & -6 \\
\hline Thalamus & & & 3.86 & -6 & -10 & -4 \\
\hline Caudate nucleus & $\mathrm{L}$ & 107 & 4.71 & -12 & 14 & 2 \\
\hline Caudate nucleus & $\mathrm{R}$ & 45 & 3.81 & 14 & 20 & 6 \\
\hline
\end{tabular}

Data are thresholded at $p<0.001$ (uncorrected), with a cluster-level of $k=10$.

a More liberal threshold for subcortical areas ( $p<0.005$ (uncorrected), $k=5$ ).

directly related to the differences in visual stimulation and the associated stimulus processing. Additionally, there were widespread bilateral clusters in the parietal cortices as well subcortical activity differences in the thalamus (extending posterior towards the colliculi but probably not laterally enough to subtend the subthalamic nucleus) and the caudate nucleus. The relative nonspecificity of this contrast, however, does not allow for a clear distinction as to which of these areas is directly related to response inhibition, or to differences in sensory processing of the Stop-stimulus.

\section{Within-SR-block conjunction contrasts of Stop- and Go-trials}

The very modest differences between successful and unsuccessful Stop-trials reported above appear to indicate that response-inhibition-related activity was present to a substantial degree in both conditions. The widespread activation differences found for the comparison between successful Stop-trials and Go-trials, on the other hand, are in accord with the view that this contrast is relatively unspecific. In an attempt to strike a compromise between these two approaches, we performed conjunction analyses that exclusively yield brain activity present in successful and unsuccessful Stop-trials, relative to a reference condition. First, in a 'within-SR-block' conjunction analysis, we used the Go-trials from the same Stoprelevant blocks as the reference condition. In frontal control areas, this contrast revealed bilateral insular, lateral frontal, and medial frontal areas (see Table 4 and Fig. 3). Furthermore, as would be expected from 
Table 4

Within-SR-block conjunction SST ('SST_SR > GT_SR' and 'UST_SR > GT_SR')

\begin{tabular}{|c|c|c|c|c|c|c|}
\hline \multirow[t]{2}{*}{ Anatomical structure } & \multirow[t]{2}{*}{ Hemisphere } & \multirow[t]{2}{*}{$\begin{array}{l}\text { Cluster size } \\
\text { [voxel] }\end{array}$} & \multirow[t]{2}{*}{$t$-value } & \multicolumn{3}{|c|}{$\begin{array}{l}\text { Peak coordinates } \\
\text { MNI }(\mathrm{mm})\end{array}$} \\
\hline & & & & $x$ & $y$ & $z$ \\
\hline \multicolumn{7}{|l|}{ Frontal cortex } \\
\hline Frontal operculum & $\mathrm{R}$ & 418 & 7.99 & 48 & 18 & 0 \\
\hline Pre-SMA & $L / R$ & 157 & 4.8 & 0 & 20 & 48 \\
\hline Pre-SMA & & & 3.74 & 8 & 16 & 60 \\
\hline Insula & $\mathrm{L}$ & 244 & 4.73 & -36 & 20 & -4 \\
\hline Frontal operculum & & & 4.12 & -46 & 18 & 8 \\
\hline Middle frontal gyrus & $\mathrm{R}$ & 36 & 4.41 & 38 & 44 & 28 \\
\hline Middle frontal gyrus & $\mathrm{L}$ & 45 & 4.24 & -34 & 40 & 30 \\
\hline \multicolumn{7}{|l|}{ Parietal and temporal cortex } \\
\hline Inferior parietal lobule & $\mathrm{R}$ & 1348 & 7.56 & 62 & -42 & 26 \\
\hline Inferior parietal lobule & & & 6.44 & 52 & -38 & 46 \\
\hline Superior temporal gyrus & & & 5.45 & 56 & -40 & 12 \\
\hline Inferior parietal lobule & $\mathrm{L}$ & 773 & 4.95 & -48 & -38 & 48 \\
\hline Superior parietal lobule & & & 4.92 & -24 & -64 & 48 \\
\hline Inferior parietal lobule & & & 4.9 & -38 & -52 & 58 \\
\hline \multicolumn{7}{|l|}{ Occipital cortex } \\
\hline Middle occipital gyrus & $\mathrm{L}$ & 696 & 7.82 & -48 & -80 & 0 \\
\hline Inferior occipital gyrus & & & 5.64 & -40 & -62 & -8 \\
\hline Middle occipital gyrus & & & 5.37 & -36 & -88 & -2 \\
\hline Inferior occipital gyrus & $\mathrm{R}$ & 751 & 6.47 & 44 & -74 & -2 \\
\hline Middle occipital gyrus & & & 5.05 & 38 & -68 & 12 \\
\hline Fusiform gyrus & & & 4.88 & 28 & -78 & -6 \\
\hline \multicolumn{7}{|l|}{ Subcortical areas ${ }^{\mathrm{a}}$} \\
\hline Anterior midbrain & $\mathrm{L} / \mathrm{R}$ & 154 & 3.77 & 0 & -12 & -4 \\
\hline Superior colliculus & & & 3.68 & 4 & -28 & -2 \\
\hline Thalamus & & & 3.24 & 4 & -18 & 0 \\
\hline Caudate nucleus & $\mathrm{L}$ & 6 & 3.24 & -8 & 16 & 6 \\
\hline
\end{tabular}

Data are thresholded at $p<0.002$ (uncorrected), with a cluster-level of $k=10$.

a More liberal threshold for subcortical areas ( $p<0.01$ (uncorrected), $k=5$ ).

the differences in physical stimulation between Stop- and Go-trials, this conjunction contrast also identified a number of areas in the ventral visual processing stream and in the parietal cortex. Subcortical activations were found in the left caudate nucleus and in a cluster comprising parts of the posterior thalamus and the superior colliculi.

Concerning the comparison with the preceding analysis that compared successful Stop-trials with Go-trials (which is one of the two contrasts that was entered into the conjunction), it is evident that fewer areas were identified. Importantly, however, the key players that have been previously related to response inhibition, such as the right IFG, the pre-SMA, and the caudate nucleus were still present in the conjunction. Using Go-trials as the reference condition, however, still has the disadvantage that it renders activity in areas that are engaged in visual stimulus processing and attention hard to interpret.

Between-block conjunction contrasts of relevant and irrelevant Stop-trials

In order to improve upon the lack of specificity in visually responsive areas in the above contrasts employing Go-trials for the comparisons, we used the "Stop"-trials from the SI-blocks (ST_SI) as the reference condition in our second conjunction analysis. This between-block conjunction of the two contrasts ('SST_SR $>$ ST_SI' and 'UST_SR > ST_SI') revealed large clusters of activity bilaterally in the inferior frontal and insular cortex, and in the right pre-SMA (see Table 5 and Fig. 4), in close accord with the frontal clusters identified by the within-SR-block conjunction. Additionally, activity was found in the thalamus, and bilaterally in the caudate nucleus. As predicted, using a reference condition matched for sensory stimulation indeed led to activation in fewer visual cortical areas. Importantly, however, a substantial cluster in the right parietal cortex, close to the temporoparietal junction (TPJ), along with a relatively small cluster in the left

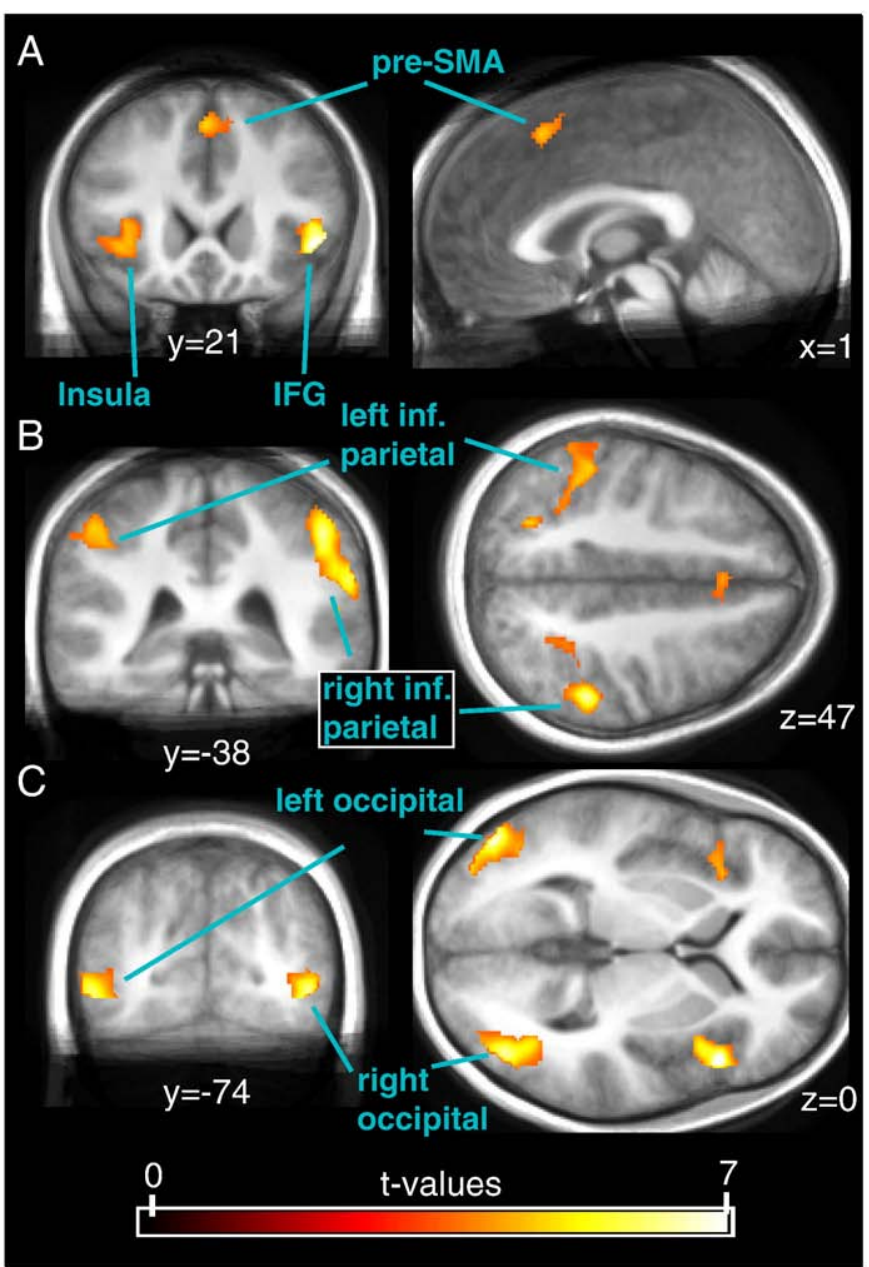

Fig. 3. Results of the within-SR-block conjunction ('SST_SR $>$ GT_SR' and 'UST_SR > GT_SR'). This conjunction, that identifies activity common to successful and unsuccessful Stop-trials as compared to Go-trials, among other areas revealed activity in the bilateral IFG/insula (A) and widespread activations in parietal (B) and occipital areas (C). The displayed data was thresholded at a $t$-value of 3.5 .

Table 5

Between-block conjunction SST ('SST_SR $>$ ST_SI' and 'UST_SR $>$ ST_SI').

\begin{tabular}{|c|c|c|c|c|c|c|}
\hline \multirow[t]{2}{*}{ Anatomical structure } & \multirow[t]{2}{*}{ Hemisphere } & \multirow[t]{2}{*}{$\begin{array}{l}\text { Cluster size } \\
\text { [voxel] }\end{array}$} & \multirow[t]{2}{*}{$t$-value } & \multicolumn{3}{|c|}{$\begin{array}{l}\text { Peak coordinates } \\
\text { MNI (mm) }\end{array}$} \\
\hline & & & & $x$ & $y$ & $z$ \\
\hline \multicolumn{7}{|l|}{ Frontal cortex } \\
\hline Frontal operculum & $\mathrm{R}$ & 243 & 7.29 & 50 & 18 & 0 \\
\hline Insula & & & 4.42 & 42 & 10 & -6 \\
\hline Insula & $\mathrm{L}$ & 187 & 4.74 & -38 & 16 & -6 \\
\hline Insula & & & 4.13 & -36 & 18 & 8 \\
\hline Insula & & & 3.7 & -30 & 22 & 2 \\
\hline Pre-SMA & $\mathrm{R}$ & 16 & 3.88 & 2 & 14 & 50 \\
\hline ACC & $\mathrm{L} / \mathrm{R}$ & 11 & 3.82 & 0 & 26 & 22 \\
\hline \multicolumn{7}{|l|}{ Parietal cortex } \\
\hline Supramarginal gyrus & $\mathrm{R}$ & 165 & 5.13 & 58 & -44 & 30 \\
\hline \multicolumn{7}{|l|}{ Occipital cortex } \\
\hline Middle occipital gyrus & $\mathrm{L}$ & 14 & 3.76 & -32 & -88 & -2 \\
\hline \multicolumn{7}{|l|}{ Subcortical areas ${ }^{\mathrm{a}}$} \\
\hline Caudate nucleus & $\mathrm{L}$ & 61 & 4.31 & -8 & 16 & 6 \\
\hline Caudate nucleus & $\mathrm{R}$ & 5 & 2.91 & 8 & 12 & 2 \\
\hline Thalamus & $\mathrm{R}$ & 50 & 3.08 & 2 & -20 & 2 \\
\hline Thalamus & $\mathrm{L}$ & & 2.73 & -2 & -12 & 0 \\
\hline
\end{tabular}

Data are thresholded at $p<0.002$ (uncorrected), with a cluster-level of $k=10$.

More liberal threshold for subcortical areas ( $p<0.01$ (uncorrected), $k=5$ ). 


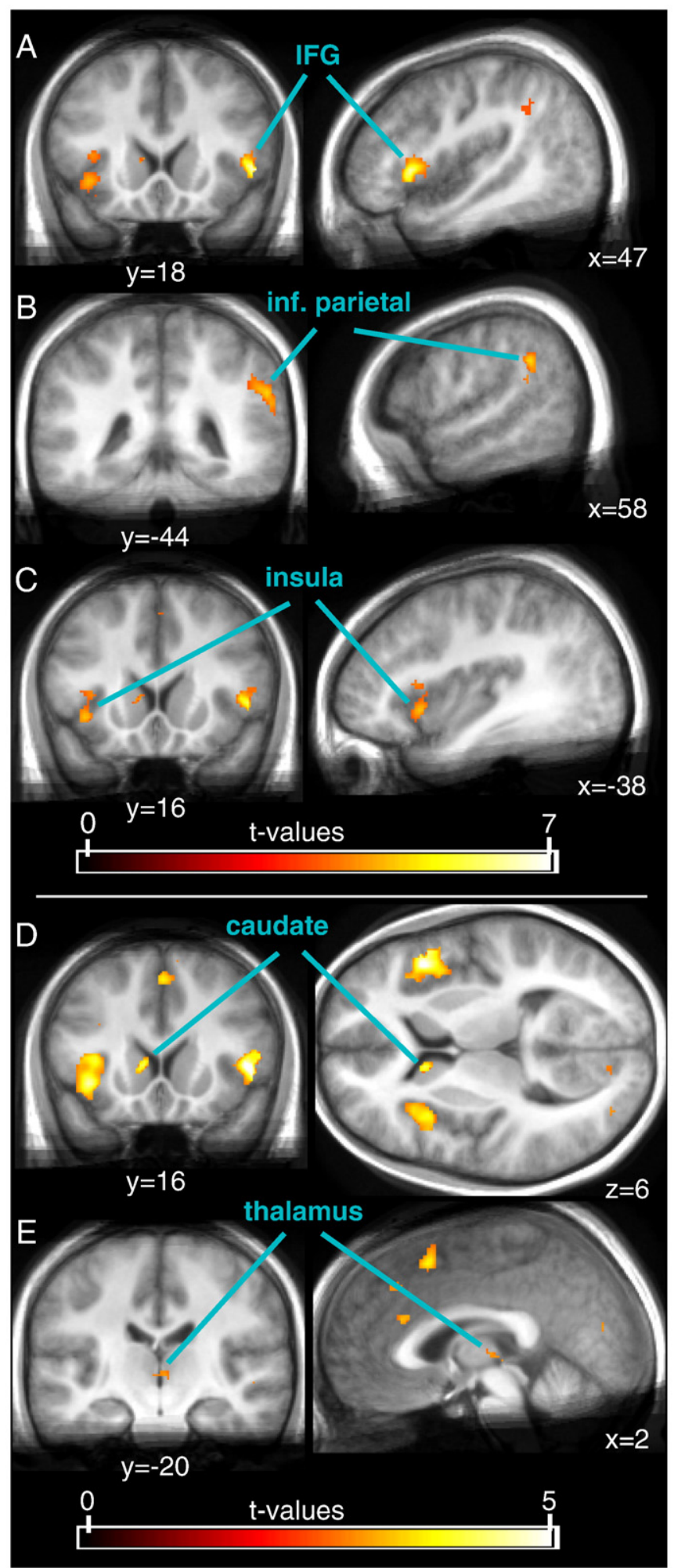

Fig. 4. Results of the between-block conjunction ('SST_SR $>$ ST_SI' and 'UST_SR $>$ ST_SI'). This conjunction, which yields activations that are in common between successful and unsuccessful Stop-trials in the Stop-relevant condition relative to "Stop"-trials in the Stop-irrelevant blocks, revealed, among other cortical areas, strong activity in the right IFG (A), right inferior parietal cortex close to the temporo-parietal junction (B), and the left insula (C). Subcortical activations were found in the caudate nucleus (D) and in the thalamus (E; note that the thalamic cluster does not extend very far laterally and ventrally and therefore seems unlikely to contain the STN). The displayed data was thresholded at a $t$-value of 3.5 in the upper three panels, and at 2.6 in the lower two. occipital lobe, was still present in this contrast. These areas therefore display stronger activations in response to Stop-stimuli when they are task-relevant, possibly reflecting enhanced attention to those stimuli.

Finally, we performed an ROI analysis within the identified clusters of the above conjunction to probe for differences between successful and unsuccessful Stop-trials. (Note that the conjunction only requires both conditions to be significantly different from the reference condition, but that they could still be different from one another). As can be seen in Fig. 5, the response to successful and unsuccessful Stop-trials was highly similar in all identified areas. A comparison of the parameter estimates in the ROIs only approached significance in the right pre-SMA ROI (UST_SR $>$ SST_SR; two-tailed $p$-value $=0.07$ ) and displayed a weak trend in the left caudate nucleus (SST_SR > UST_SR; two-tailed $p$-value $=0.16$; all other $p>0.25$ ). This further suggests that the two conditions are highly similar in the areas identified by the conjunction.

\section{Across-subject correlation with SSRT}

One common way to demonstrate that a given brain area is directly involved in response inhibition in the Stop-signal task has been to show that its activity differs between fast and slow inhibitors (i.e., short and long SSRTs; Aron et al., 2007a; Aron and Poldrack, 2006; Li et al., 2006; Rubia et al., 2007; Zheng et al., 2008). To this end, we performed a correlational analysis using the activity estimates in the thirteen local maxima identified by the between-block conjunction (see Table 5). We hypothesized that we would find negative correlations with the SSRT values (Bonferroni-corrected $p$-value corresponding to an overall alpha-level of 0.05 for 13 tests: 0.004 ) in areas that are especially relevant for response inhibition, suggesting their stronger involvement in subjects with better inhibition performance. Such a negative correlation could only be found in one of the thirteen areas, however, namely the left anterior insula (at the local maximum at MNI $(x, y, z)=-38,16,-6$; Pearson's $r=-0.69$; $p=0.002$; see Fig. $6 \mathrm{~A})$. None of the other areas displayed significant correlations with SSRT (all $p>0.05$ ).

In order to estimate the specificity of this area's role in stopping, we additionally correlated activity estimates from this area with response times (for Go-trials from both task blocks, unsuccessful Stop-trials from SR-blocks, and "Stop"-trials from SI-blocks) and with task accuracy (for all trial types where subjects were always supposed to respond - i.e., Go-trials from both blocks and "Stop"trials from SI-blocks). We hypothesized that relevant areas would display negative correlations with RT and positive correlations with accuracy (Bonferroni-corrected $p$-value for 7 tests: 0.008). Correlations with RT reached even the uncorrected significance level only for the "Stop"-trials from the SI-blocks $(r=-0.47 ; p=0.04)$, although all correlations with RT were consistently negative (all other $p>0.1$ ). However, correlations with accuracy rate for Go-trials from SR-blocks and Stop-trials from SI-blocks were highly significant $(r=0.66$, $p=0.004 ; r=0.72 ; p=0.001$; Fig. $6 \mathrm{~B})$, whereas the correlation with accuracy in Go-trials from SI-blocks was only significant on an uncorrected significance level $(r=0.47 ; p=0.04)$. Thus it appears that a common underlying factor that affects task performance relatively globally influences SSRTs and task accuracy across subjects, whereas it does not appear to affect overall response times very much. Consistent with this idea, a third correlational analysis between the three accuracy measures and SSRT found strong negative relationships (SSRT with accuracy in: SR-block Go-trials $(r=-0.81 ; p=0.001)$, SI-block Go-trials $(r=-0.5 ; p=0.03)$, and SI-block "Stop"-trials $(r=-0.77 ; p=0.001)$; Fig. $6 C)$.

\section{Comparing Go-trials from SR- and SI-blocks}

It is well known that in the Stop-signal task subjects tend to proactively (i.e., strategically) slow down motor responses to the choice- 


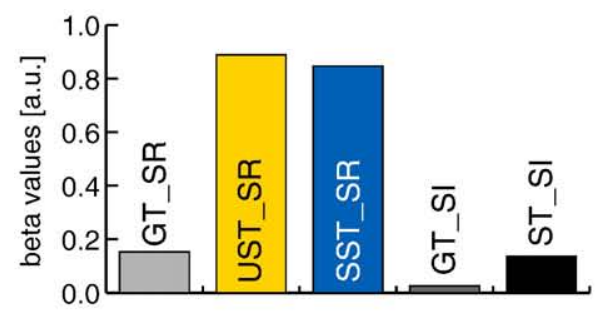

right inferior frontal

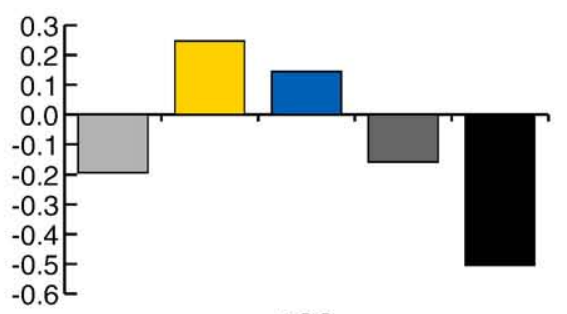

ACC

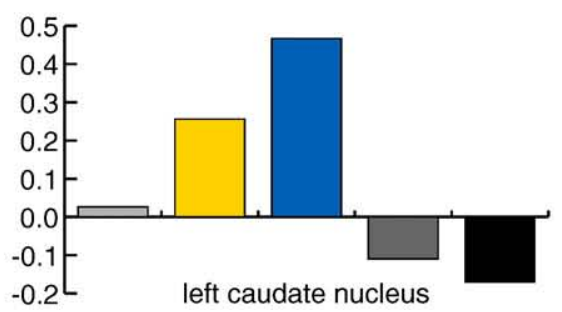

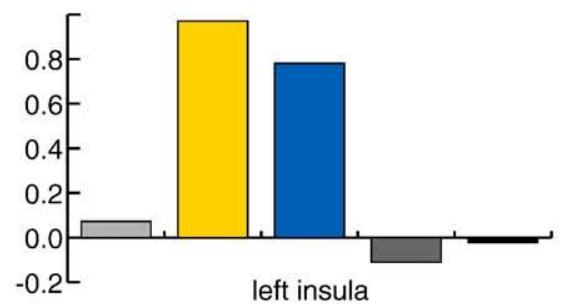

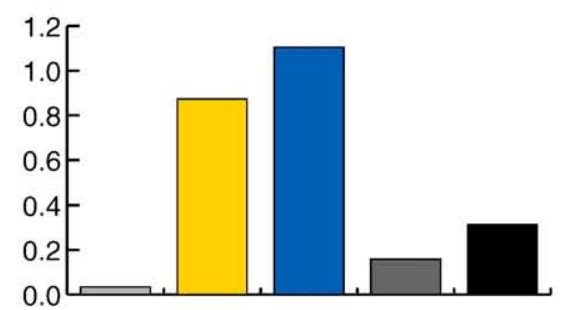

right supramarginal gyrus

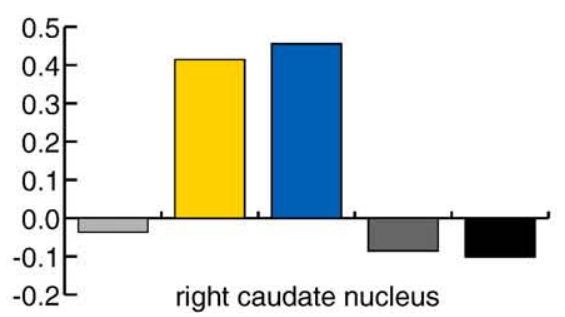

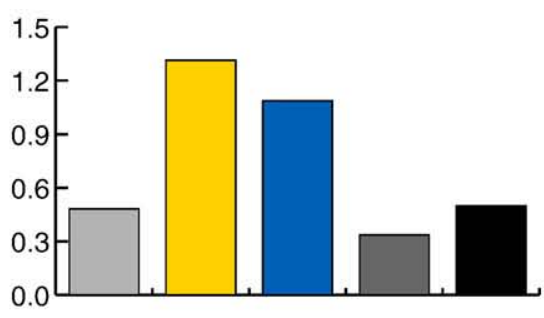

right pre-SMA

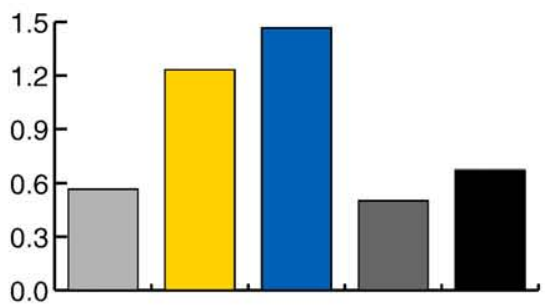

left occipital gyrus

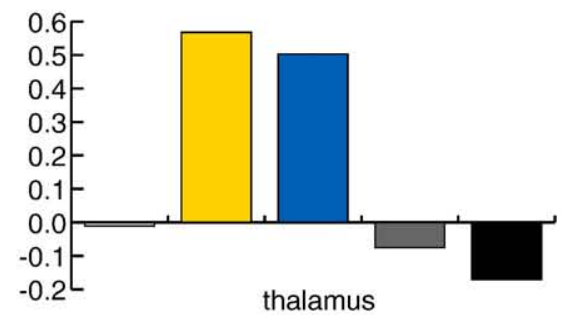

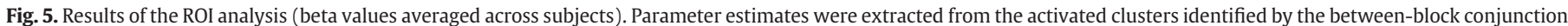

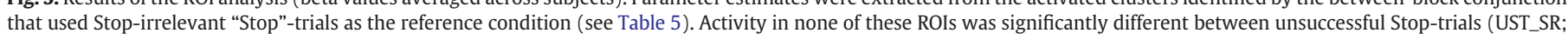
yellow bars) and successful Stop-trials (SST_SR; blue bars).

reaction stimulus when a relevant Stop-stimulus might follow (also referred to as "withholding"; Chevrier et al., 2007; Jaffard et al., 2008; Verbruggen and Logan, 2009). Such withholding was apparent in our behavioral data, as seen by the Go-trial RTs in the Stop-relevant task blocks being $84 \mathrm{~ms}$ slower than Go-trial RTs in the Stop-irrelevant task blocks. To investigate activity related to this process that may have potentially inflated the results of the between-block conjunction, we directly compared the Go-trial conditions from the two task blocks.
The contrast of SR-block Go-trials versus SI-block Go-trials yielded activity only in a small cluster in the vicinity of (slightly below) the left striatum (max. $t$-value: 3.69 ; peak MNI coordinates: $-4,14,-10$; cluster size (at $p<0.005)$ : 5 ). Since this contrast only differs in the task context (SR-block vs. SI-block) this activity may be related to the pro-active slowing of responses during all trials in the SR-blocks. Regardless, however, activity differences between Go-trials under the two different instructions were rather slight in the present study.
A

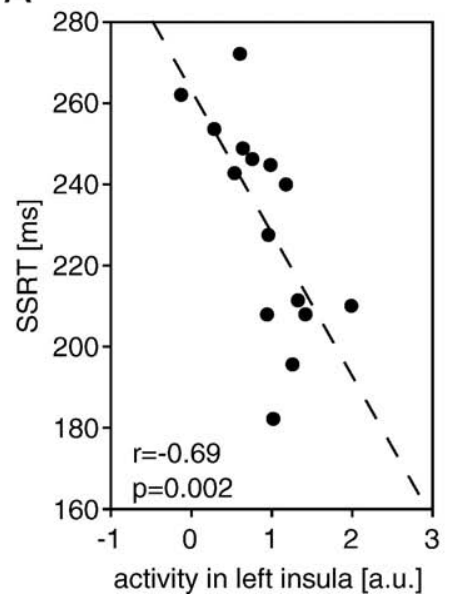

B

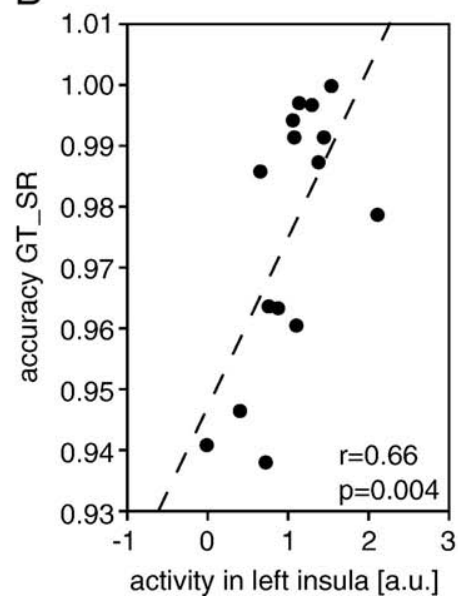

C

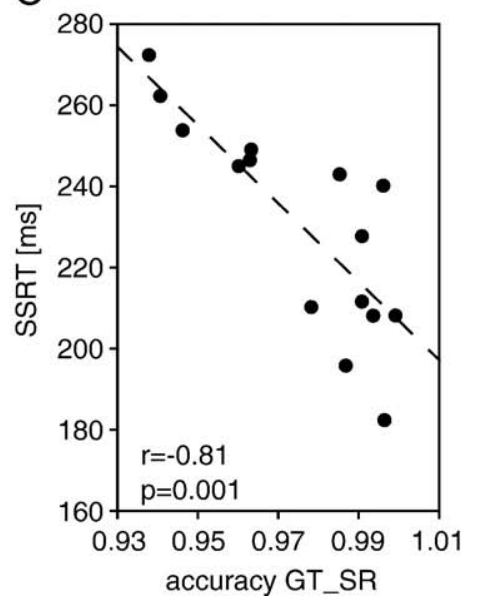

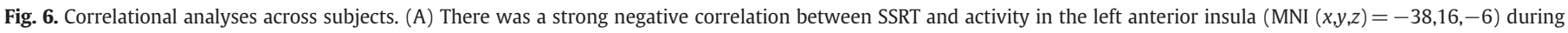

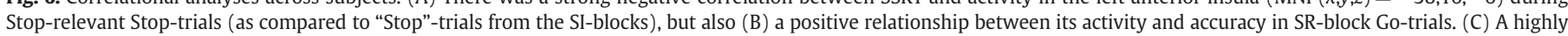
significant negative correlation was furthermore found directly between SSRT and SR-block Go-trial accuracy. 


\section{A 4-way conjunction (second-level)}

In order to further rule out that general block differences between SR- and SI-blocks inflated the results of the between-block conjunction, a final analysis was performed combining the two preceding conjunction analyses (i.e., a second-level conjunction analysis between the within-SR-block conjunction and the between-block conjunction; see supplementary Table S2). Consistent with the idea that the between-block conjunction (using the sensory reference condition) essentially yields a subgroup of the activations of the within-SR-block conjunction (using Go-trials as a reference condition), the conjunction of the two brought nearly identical results as the between-block conjunction alone. The only difference appeared to be slightly smaller cluster sizes, and somewhat reduced activity in the caudate nucleus, consistent with its lower activation level in the within-SR-block conjunction.

\section{Within-SR-block conjunction investigating unsuccessful Stop-trials}

While the focus of the present work lies on identifying areas that are involved in response inhibition, the conjunction approach suggested here extends nicely to processes related to unsuccessful stopping, too. These processes might have to do with the hastiness with which a response is given, monitoring of the outcome, and other functions. To characterize such processes, earlier approaches have typically compared unsuccessful Stop-trials either with successful Stop-trials (e.g., Li et al., 2006) or Go-trials (e.g., Rubia et al., 2003). Both of these direct contrasts, however, are somewhat limited in that the first compares conditions with an overt response versus without one, whereas the second differs greatly in sensory stimulation. Performing these contrasts on our data (see supplementary Tables S3 and S4) revealed results that are consistent with this notion, including motor areas and sensory areas respectively. Conjoining those two contrasts, however, resulted in a much more clear-cut result pattern, with activations primarily in the medial frontal cortex and the thalamus (Table 6).

\section{Discussion}

The present fMRI study investigated neural processes underlying response inhibition during the Stop-signal task in human subjects. We have argued that some of the heterogeneity between results from preceding fMRI studies may have arisen from the use of different functional contrasts and that these prior approaches may have either lacked some specificity or were overly conservative concerning

Table 6

Within-block conjunction UST ('UST_SR > SST_SR' and 'UST_SR>GT_SR').

\begin{tabular}{|c|c|c|c|c|c|c|}
\hline \multirow[t]{2}{*}{ Anatomical structure } & \multirow[t]{2}{*}{ Hemisphere } & \multirow[t]{2}{*}{$\begin{array}{l}\text { Cluster size } \\
\text { [voxel] }\end{array}$} & \multirow[t]{2}{*}{$t$-value } & \multicolumn{3}{|c|}{$\begin{array}{l}\text { Peak coordinates } \\
\text { MNI (mm) }\end{array}$} \\
\hline & & & & $x$ & $y$ & $z$ \\
\hline \multicolumn{7}{|l|}{ Frontal cortex } \\
\hline Dorsal ACC & $\mathrm{R}$ & 39 & 4.91 & 6 & 26 & 38 \\
\hline Pre-SMA & $\mathrm{L} / \mathrm{R}$ & 27 & 3.92 & 0 & 16 & 44 \\
\hline ACC & $\mathrm{R}$ & 29 & 3.86 & 2 & 48 & 26 \\
\hline \multicolumn{7}{|l|}{ Parietal cortex } \\
\hline Mid-cingulate cortex & $\mathrm{R}$ & 74 & 4.68 & 4 & -20 & 32 \\
\hline $\begin{array}{l}\text { Posterior cingulate } \\
\text { cortex }\end{array}$ & $\mathrm{R}$ & 19 & 3.95 & 4 & -38 & 24 \\
\hline \multicolumn{7}{|l|}{ Subcortical areas ${ }^{\mathrm{a}}$} \\
\hline Thalamus & $\mathrm{L}$ & 446 & 5.32 & -8 & -18 & 8 \\
\hline Superior colliculus & $\mathrm{R}$ & & 4.44 & 8 & -38 & -6 \\
\hline
\end{tabular}

Data are thresholded at $p<0.002$ (uncorrected), with a cluster-level of $k=10$. ${ }^{(a)}$ More liberal threshold for subcortical areas ( $p<0.01$ (uncorrected), $k=5$ ). response-inhibition-related brain activity. Here we have suggested using conjunction analyses of both Stop-trial types against a reference condition as a compromise between the two earlier approaches. Such conjunction contrasts are valid using either Go-trials as a reference condition (at the cost of limiting the ability to interpret visually responsive areas due to the visual sensory differences) or a control condition matched for physical stimulation (such as the Stopirrelevant sensory control condition employed here).

We have demonstrated that this approach identifies a relatively clear-cut network of brain areas discussed in contemporary neuroanatomical models of response inhibition, including right inferior prefrontal and medial frontal areas, as well as parts of the basal ganglia and the thalamus. Additionally, areas related to the sensory processing of the Stop-stimulus (in occipital cortex) and potential sources of attentional modulations thereof (right supramarginal gyrus) were identified as being more active during task blocks where Stop-stimuli were relevant versus not relevant. The small number of areas that were identified by the respective contrasts but were not part of the typical areas found in Stop-signal tasks has to be interpreted with care, in that their occurrence was not predicted a priori. Since we did not apply corrected significance thresholds, those activations could represent type-I errors. Finally, we found that subjects that inhibit their responses faster than others (i.e., have shorter SSRTs) display stronger activity in the left anterior insula. This correlation, however, appeared not to be specifically linked to response inhibition alone, as the activity in this area also correlated very robustly with general accuracy measures, which in turn was also directly correlated with the SSRT.

\section{Comparison of different analysis strategies}

The logic of the present experiment was to identify brain areas that are active in both successful and unsuccessful Stop-trials, while excluding activations that are specific to either condition. The direct comparison between successful and unsuccessful Stop-trials yielded only a few, relatively weakly activated, areas, suggesting that response-inhibition-related activity was present to a similar degree in both conditions. This general similarity was furthermore underscored by an ROI analysis comparing successful and unsuccessful Stop-trials directly within the areas identified by the between-block conjunction contrast that did not find any significant differences. The conjunction logic exploits this trait to identify neural activity related to this function in a way that is less conservative and yet still relatively specific. Related approaches usually combined data from different experiments, and have been employed to identify a common network underlying different variants of the Stop-signal task (Aron et al., 2007a; Coxon et al., 2009; Leung and Cai, 2007; Xue et al., 2008) or to investigate the overlap with results from go/no-go tasks (Rubia et al., 2001; Zheng et al., 2008). Heretofore, however, no study has employed the conjunction logic to identify activity that is common to successful and unsuccessful Stop-trials relative to a reference condition. Using a comparable approach for unsuccessful Stop-trials both compared with Go-trials and successful Stop-trials also appeared to provide more specific results than both of these individual contrasts.

Evaluating the performance of the direct comparison between successful and unsuccessful Stop-trials in our data, the generally small number of activated areas (a small cluster in occipital cortex, the IFG, and the caudate nucleus) argues in favor of our notion that this contrast was very conservative in estimating response-inhibitionrelated activity. Moreover, even for these few areas it is not clear whether they were directly related to response inhibition. Activity in visual cortex may well reflect differences in how well the Stopstimulus is attended in successful vs. unsuccessful Stop-trials (see below). The frontal cluster, on the other hand, appeared to be somewhat more dorsal and posterior than the areas identified by the 
conjunction contrasts and by earlier work, so that it is hard to determine whether the underlying cognitive/neural operation is directly implicated in response inhibition or not. Finally, stronger activity for successful than for unsuccessful Stop-trials was found in the caudate nucleus, which might indeed be related to response inhibition (Li et al., 2008; Rubia et al., 2007; Vink et al., 2005). Alternatively, as has been argued previously (Aron and Poldrack, 2006), it could signify stronger pro-active response slowing (slower responding to the Go-stimulus already before the Stop-stimulus is even presented) during successful Stop-trials, a function that has also been attributed to the caudate nucleus (Vink et al., 2005).

Furthermore, the small amount of differences between successful and unsuccessful Stop-trials also points in the direction that there is only a small number of processes that are exclusively present (or clearly more pronounced) in successful Stop-trials. This might indicate that earlier studies employing the approach of comparing successful Stop-trials vs. Go-trials likely obtained reasonable estimates of response-inhibition-related activity in frontal and subcortical control regions. Regardless, the direct, albeit qualitative, comparison between that approach and the conjunction analyses in the present data attributes more specificity to the conjunction approach. Moreover, using the conjunction between successful and unsuccessful Stop-trials allowed us to define functional ROIs in a way that was not biased to either condition, thus permitting their subsequent comparison (Kriegeskorte et al., 2009). Conversely, both the direct comparison of successful vs. unsuccessful Stop-trials or of successful Stop-trials vs. Gotrials would bias the results of such an ROI analysis in favor of successful Stop-trials.

\section{The neural basis of response inhibition}

All of the conjunction contrasts performed here identified a network of mostly cortical areas that included key players of the responseinhibition network that have been suggested based on earlier work (for recent reviews see Aron et al., 2007b; Chambers et al., 2009; Verbruggen and Logan, 2008). This clearly speaks to the notion that the conjunction contrasts, although perhaps somewhat less sensitive than the direct comparison between successful Stop-trials and Gotrials, were more specific than approaches using Go-trials as a reference condition. All conjunction contrasts found strong activity in the right frontal operculum extending into the anterior insula and activity in the right pre-SMA (e.g., Aron et al., 2007a; Aron and Poldrack, 2006; Rubia et al., 2003; Rubia et al., 2007; Xue et al., 2008). Subcortically, we found evidence for an involvement of the caudate nucleus in stopping, but failed to find significant activity in the subthalamic nucleus. It has been argued that responses can be either cancelled by (re)activating the indirect route to the striatum (i.e. caudate nucleus and putamen), or by way of the hyperdirect route from control regions to the STN (Aron et al., 2007a; Aron and Poldrack, 2006; Eagle et al., 2008; Nambu et al., 2002; Ray et al., 2009; Vink et al., 2005). Although there is no convincing evidence for an STN involvement in our data, whereas activity in the caudate nucleus was found for Stop-trials, we do not want to conclude that this is evidence against a role for the hyperdirect route. Specifically, the STN is an extremely small structure which poses challenges for fMRI, particularly for whole-head scans (Aron and Poldrack, 2006). Thus, it could well be that the present experiment simply did not have the sensitivity to identify activity in this region.

\section{The role of visual attention in response inhibition}

A further goal of the present study was to examine the role of occipital, temporal, and parietal effects seen in the Stop-signal task in one of the standard analysis strategies that has been employed, namely the comparison of Stop- and to Go-trials. EEG and MEG work has indicated that the strength of the sensory processing of the taskrelevant stimuli, especially the Stop-stimulus, influences whether or not stopping is successful (Bekker et al., 2005; Boehler et al., 2009; Knyazev et al., 2008). This has been taken to indicate that more effective processing of the Stop-stimulus might speed up or otherwise facilitate the Stop-process, consequently enhancing the probability of catching up with the Go-process before a response is executed. The comparison between successful and unsuccessful Stop-trials in the present experiment appears to provide evidence consistent with this notion, in that there was slightly stronger activity in an area of the occipital cortex during successful stopping. However, the difference was very small, so that this finding would need to be replicated to be certain.

On a more global level, we compared the Stop-trials from trial blocks where the Stop-stimuli were task-relevant, versus when they were not. Previous studies using comparisons between Stop-trials and Go-trials commonly identified large clusters of activity in areas of the ventral visual processing stream, as well as substantial clusters throughout the parietal cortices (e.g., Aron and Poldrack, 2006; Coxon et al., 2009). Due to the clear difference in visual stimulation, however, these studies were largely hampered in their interpretations of these activations. Particularly the parietal cortex may or may not subserve an integral and direct role in response inhibition (Bunge et al., 2002; Chambers et al., 2009; Coxon et al., 2009; Wager et al., 2005). Alternatively, these areas could simply be activated by the second stimulus either in a merely sensory fashion or by attentional capture to the infrequent Stop-stimuli (Yantis, 1993).

In the present data, in the contrast between Stop-trials in the Stop-relevant blocks versus those in the Stop-irrelevant blocks, stronger activity was observed in an occipital area that is consistent with being a mid-level visual processing area. This difference, however, was relatively modest. Much stronger activity differences were found in the right inferior parietal cortex in the vicinity of the temporo-parietal junction. This result pattern appears to be in accord with an earlier EEG study that also found stronger activity over occipito-parietal cortex to Stop-stimuli in trial blocks where they were task-relevant versus when they were task-irrelevant (Schmajuk et al., 2006). This difference in parietal cortex activity might indicate stronger capturing of attentional resources during Stop-trials in Stop-relevant blocks, which fits with the notion that bottom-up attentional recruitment does interact with stimulus relevance (Folk et al., 1992; Yantis and Jonides, 1990), and that the inferior parietal cortex is a key player in this function (Corbetta and Shulman, 2002). Importantly no such difference was found between the Go-trials in the two trial blocks, consistent with the fact that Go-stimuli were task-relevant under both task regimes. Beyond this right inferior parietal cortex activation, however, the present data did not identify any other area in parietal cortex that appeared to have a more direct involvement in response inhibition, such as through direct thalamocortical interactions originating from parietal cortex (Wager et al., 2005).

\section{Between-subject correlations}

Multiple groups have argued that between-subject differences in SSRT should correlate inversely with the activity in brain areas that are particularly important for response inhibition (Aron and Poldrack, 2006; Li et al., 2006, 2008; Rubia et al., 2007; Zheng et al., 2008, for a report of a positive correlation). However, the results of previous studies along these lines have not been consistent. One study found such correlations in the right IFG and STN (Aron and Poldrack, 2006), another in superior frontal cortex and the left pre-central gyrus (Li et al., 2006, 2008), and a study investigating development of inhibitory function across different ages found a relationship between SSRT and activity in bilateral inferior frontal gyrus, in the thalamus, the cerebellum, and the striatum (Rubia et al., 2007). The present study identified yet another region that correlated negatively with 
SSRT, the left anterior insula, whereas none of the aforementioned correlations was replicated in the present data. ${ }^{3}$

Contrary to earlier findings, however, it would appear that the correlation with SSRTs in our experiment was not specifically indicative of differences in stopping efficacy, as it turned out that activity in the left anterior insula also correlated with general task accuracy. Furthermore SSRTs directly correlated with general task accuracy as well, suggesting a common underlying cause for both variables - a finding that, to our knowledge, has not been reported before. Notably, this underlying cause appeared to be very stable across different experimental conditions, as significant correlations were not only present for measures taken in the same trial Stop-relevant trial blocks (SR), but also in the SI-blocks. We therefore suggest that activity in the left anterior insula may reflect a mechanism influencing the general level of task performance rather than exclusively being related to stopping efficacy. The anterior insula has been suggested to play a central role in maintaining stable task set control (e.g., Dosenbach et al., 2008) and therefore may be related to the subjects' motivation and/or ability to maintain high levels of task control.

In general, it appears that correlations between SSRT and task accuracy should be explored when using between-subject differences in SSRT to link activity in a certain brain area to response inhibition. Aron and Poldrack (2006), for example, reported that there was no correlation with Go-trial RT in the areas that correlated with SSRT in their study. However, RTs might be the result of multiple mechanisms, especially in the presence of a tracking algorithm that keeps stopping performance in the same range for different response strategies (e.g., pro-active response slowing). Therefore, RTs might not reflect general task performance as reliably as task accuracy. In Li et al. (2006), on the other hand, a comparison was performed between two groups of subjects with fast vs. slow SSRTs, and the two groups did not differ in Go-trial accuracy. Possible explanations for this difference with the present data could be related to the specific paradigm or features inherent to accuracy measures that tend to be affected by the proximity to the performance ceiling.

On a simple behavioral level, our finding that the SSRT is not independent from task accuracy would suggest a possible problem with the SSRT's typical interpretation. The SSRT has been used extensively to characterize response inhibition, which has been of particular interest in studies investigating human development as well as pathologies like ADHD or drug abuse, where inhibitory control is a central concept (e.g., Barkley, 1997) and appears to be affected relatively specifically (Groman et al., 2009; Williams et al., 1999). While a wealth of experimental investigations has demonstrated many favorable features of the SSRT, like its immunity against response strategies (e.g., slowing down Go-stimulus responses), the present finding calls into question whether differences in SSRT indeed distinctively reflect variable degrees of inhibitory motor control. Interestingly, some recent investigations of the Stop-signal task in ADHD populations as compared to controls point in the direction that prolonged SSRTs in children with ADHD might indeed not reflect deficits in inhibitory motor control but rather slower processing of both the Go- and the Stop-stimuli (Alderson et al., 2007, 2008; Lijffijt et al., 2005).

\section{Neural processes related to unsuccessful stopping}

In the present paper, we also extended our conjunction approach to the investigation of processes related to unsuccessful stopping.

\footnotetext{
${ }^{3}$ It should be emphasized that we adopted a fairly simple approach to performing the brain-behavior analysis. Specifically, we extracted activity estimates only from areas that were identified by the between-block conjunction. Therefore, these analyses could only identify brain-behavior relationships within this set of areas. Moreover, the relatively limited number of subjects here $(n=15)$ might have restricted our ability to detect further correlational relationships on a between-subject basis.
}

Previous studies have compared unsuccessful Stop-trials either with successful Stop-trials (e.g., Li et al., 2006) or Go-trials (e.g., Rubia et al., 2003). Both contrasts have clear disadvantages in that the first compares conditions with and without an overt response, whereas the second differs greatly in sensory stimulation and some additional aspects common to Stop-trials. Conjoining the two contrasts, however, should ameliorate both of these problems, and such an approach yielded a relatively circumscribed network of medial frontal and posterior cingulate areas plus the left thalamus. Activity in medial frontal areas probably reflects error detection, which has been reported for the Stop-signal task before (e.g., Li et al., 2006; Rubia et al., 2003), as has been stronger activity in the posterior cingulate cortex for unsuccessful Stop-trials (e.g., Rubia et al., 2007). These findings receive further credibility by our replication in a conjunction approach that is relatively well matched both for motor components (unsuccessful Stop-trials vs. Go-trials), as well as sensory stimulation, response inhibition, and general performance monitoring (unsuccessful vs. successful Stop-trials).

\section{Summary and conclusions}

We have argued that earlier approaches aimed at identifying activity that is related to response inhibition in fMRI studies were limited in that they have tended to be overly conservative or relatively unspecific. We have demonstrated that employing conjunctions of successful and unsuccessful Stop-trials respectively against a reference condition can provide a viable compromise that engenders a reasonable degree of specificity without being overly conservative. The most drastic increase in specificity here, however, was achieved by integrating a sensory control condition that separates out effects related to differences in sensory stimulation between Stop-trials and Go-trials. This allowed us to better interpret the resulting circumscribed activations in occipital and parietal cortex that are likely related to enhanced attention to the Stop-stimulus when it is taskrelevant. Finally, we have demonstrated that the SSRT is correlated across subjects with activity in the left anterior insula. Notably, however, activity in this area also correlated with general task accuracy, which was furthermore directly correlated with SSRT. Accordingly, this area did not appear to be directly implicated in response inhibition but rather in keeping up high levels of task performance more generally, thus calling into question the specificity with which differences in SSRT between subjects reflect differential response-inhibition abilities.

\section{Acknowledgments}

This research was supported by NIH grants RO1-MH060415 and R01-NS051048 to M.G.W. and funds of the Deutsche Forschungsgemeinschaft (BO 3345/1-1) to C.N.B.

\section{Appendix A. Supplementary data}

Supplementary data associated with this article can be found, in the online version, at doi:10.1016/j.neuroimage.2010.04.276.

\section{References}

Alderson, R.M., Rapport, M.D., Kofler, M.J., 2007. Attention-deficit/hyperactivity disorder and behavioral inhibition: a meta-analytic review of the stop-signal paradigm. J. Abnorm. Child Psychol. 35, 745-758.

Alderson, R.M., Rapport, M.D., Sarver, D.E., Kofler, M.J., 2008. ADHD and behavioral inhibition: a re-examination of the stop-signal task. J. Abnorm. Child Psychol. 36, 989-998.

Aron, A.R., 2009. Introducing a special issue on stopping action and cognition. Neurosci. Biobehav. Rev. 33, 611-612.

Aron, A.R., Behrens, T.E., Smith, S., Frank, M.J., Poldrack, R.A., 2007a. Triangulating a cognitive control network using diffusion-weighted magnetic resonance imaging (MRI) and functional MRI. J. Neurosci. 27, 3743-3752. 
Aron, A.R., Durston, S., Eagle, D.M., Logan, G.D., Stinear, C.M., Stuphorn, V., 2007b. Converging evidence for a fronto-basal-ganglia network for inhibitory control of action and cognition. J. Neurosci. 27, 11860-11864.

Aron, A.R., Poldrack, R.A., 2006. Cortical and subcortical contributions to Stop signal response inhibition: role of the subthalamic nucleus. J. Neurosci. 26, 2424-2433.

Barkley, R.A., 1997. Behavioral inhibition, sustained attention, and executive functions: constructing a unifying theory of ADHD. Psychol. Bull. 121, 65-94.

Bekker, E.M., Kenemans, J.L., Hoeksma, M.R., Talsma, D., Verbaten, M.N., 2005. The pure electrophysiology of stopping. Int. J. Psychophysiol. 55, 191-198.

Boehler, C.N., Munte, T.F., Krebs, R.M., Heinze, H.J., Schoenfeld, M.A., Hopf, J.M., 2009 Sensory MEG responses predict successful and failed inhibition in a stop-signal task. Cereb. Cortex 19, 134-145.

Boucher, L., Palmeri, T.J., Logan, G.D., Schall, J.D., 2007. Inhibitory control in mind and brain: an interactive race model of countermanding saccades. Psychol. Rev. 114, 376-397.

Bunge, S.A., Hazeltine, E., Scanlon, M.D., Rosen, A.C., Gabrieli, J.D., 2002. Dissociable contributions of prefrontal and parietal cortices to response selection. Neuroimage $17,1562-1571$.

Chambers, C.D., Garavan, H., Bellgrove, M.A., 2009. Insights into the neural basis of response inhibition from cognitive and clinical neuroscience. Neurosci. Biobehav. Rev. 33, 631-646.

Chevrier, A.D., Noseworthy, M.D., Schachar, R., 2007. Dissociation of response inhibition and performance monitoring in the stop signal task using event-related fMRI. Hum. Brain Mapp. 28, 1347-1358.

Corbetta, M., Shulman, G.L., 2002. Control of goal-directed and stimulus-driven attention in the brain. Nat. Rev. Neurosci. 3, 201-215.

Coxon, J.P., Stinear, C.M., Byblow, W.D., 2009. Stop and go: the neural basis of selective movement prevention. J. Cogn. Neurosci. 21, 1193-1203.

de Jong, R., Coles, M.G.H., Logan, G.D., Gratton, G., 1990. Searching for the point of no return: the control of response processes in speeded choice reaction performance. J. Exp. Psychol. Hum. Percept. Perform. 16, 164-184.

Dosenbach, N.U., Fair, D.A., Cohen, A.L., Schlaggar, B.L., Petersen, S.E., 2008. A dualnetworks architecture of top-down control. Trends Cogn. Sci. 12, 99-105.

Duann, J.R., Ide, J.S., Luo, X., Li, C.S., 2009. Functional connectivity delineates distinct roles of the inferior frontal cortex and presupplementary motor area in stop signal inhibition. J. Neurosci. 29, 10171-10179.

Eagle, D.M., Baunez, C., Hutcheson, D.M., Lehmann, O., Shah, A.P., Robbins, T.W., 2008 Stop-signal reaction-time task performance: role of prefrontal cortex and subthalamic nucleus. Cereb. Cortex 18, 178-188.

Folk, C.L., Remington, R.W., Johnston, J.C., 1992. Involuntary covert orienting is contingent on attentional control settings. J. Exp. Psychol. Hum. Percept. Perform. $18,1030-1044$.

Friston, K.J., Fletcher, P., Josephs, O., Holmes, A., Rugg, M.D., Turner, R., 1998. Eventrelated fMRI: characterizing differential responses. Neuroimage 7, 30-40.

Friston, K.J., Penny, W.D., Glaser, D.E., 2005. Conjunction revisited. Neuroimage 25 661-667.

Garavan, H., Ross, T.J., Murphy, K., Roche, R.A., Stein, E.A., 2002. Dissociable executive functions in the dynamic control of behavior: inhibition, error detection, and correction. Neuroimage 17, 1820-1829.

Groman, S.M., James, A.S., Jentsch, J.D., 2009. Poor response inhibition: at the nexus between substance abuse and attention deficit/hyperactivity disorder. Neurosci. Biobehav. Rev. 33, 690-698.

Hinrichs, H., Scholz, M., Tempelmann, C., Woldorff, M.G., Dale, A.M., Heinze, H.J., 2000. Deconvolution of event-related fMRI responses in fast-rate experimental designs: tracking amplitude variations. J. Cogn. Neurosci. 12 (Suppl 2), 76-89.

Jaffard, M., Longcamp, M., Velay, J.L., Anton, J.L., Roth, M., Nazarian, B., Boulinguez, P., 2008. Proactive inhibitory control of movement assessed by event-related fMRI. Neuroimage 42, 1196-1206.

Knyazev, G.G., Levin, E.A., Savostyanov, A.N., 2008. A failure to stop and attention fluctuations: an evoked oscillations study of the stop-signal paradigm. Clin. Neurophysiol. 119, 556-567.

Kriegeskorte, N., Simmons, W.K., Bellgowan, P.S., Baker, C.I., 2009. Circular analysis in systems neuroscience: the dangers of double dipping. Nat. Neurosci. 12, 535-540.

Leung, H.-C., Cai, W., 2007. Common and differential ventrolateral prefrontal activity during inhibition of hand and eye movements. J. Neurosci. 27, 9893-9900.

Li, C.S., Huang, C., Constable, R.T., Sinha, R., 2006. Imaging response inhibition in a stopsignal task: neural correlates independent of signal monitoring and post-response processing. J. Neurosci. 26, 186-192.
Li, C.S., Yan, P., Sinha, R., Lee, T.W., 2008. Subcortical processes of motor response inhibition during a stop signal task. Neuroimage 41, 1352-1363.

Lijffijt, M., Kenemans, J.L., Verbaten, M.N., van Engeland, H., 2005. A meta-analytic review of stopping performance in attention-deficit/hyperactivity disorder: deficient inhibitory motor control? J. Abnorm. Psychol. 114, 216-222.

Logan, G.D., 1994. On the ability to inhibit thought and action: a user's guide to the stop signal paradigm. In: Dagenbach, D., Carr, T.H. (Eds.), Inhibitory Processes in Attention, Memory, and Language. Academic Press, San Diego, pp. 189-239.

Logan, G.D., Cowan, W.B., 1984. On the ability to inhibit thought and action: a theory of an act of control. Psychol. Rev. 91, 295-327.

Logan, G.D., Cowan, W.B., Davis, K.A., 1984. On the ability to inhibit simple and choice reaction time responses: a model and a method. J. Exp. Psychol. Hum. Percept. Perform. 10, 276-291.

Menon, V., Adleman, N.E., White, C.D., Glover, G.H., Reiss, A.L., 2001. Error-related brain activation during a Go/NoGo response inhibition task. Hum. Brain Mapp. 12, 131-143.

Mink, J.W., 1996. The basal ganglia: focused selection and inhibition of competing motor programs. Prog. Neurobiol. 50, 381-425.

Nambu, A., Tokuno, H., Takada, M., 2002. Functional significance of the corticosubthalamo-pallidal 'hyperdirect' pathway. Neurosci. Res. 43, 111-117.

Nichols, T., Brett, M., Andersson, J., Wager, T., Poline, J.B., 2005. Valid conjunction inference with the minimum statistic. Neuroimage 25, 653-660.

Pliszka, S.R., Glahn, D.C., Semrud-Clikeman, M., Franklin, C., Perez 3rd, R., Xiong, J., Liotti, M., 2006. Neuroimaging of inhibitory control areas in children with attention deficit hyperactivity disorder who were treatment naive or in long-term treatment Am. J. Psychiatry 163, 1052-1060.

Ramautar, J.R., Slagter, H.A., Kok, A., Ridderinkhof, K.R., 2006. Probability effects in the stop-signal paradigm: the insula and the significance of failed inhibition. Brain Res. 1105, 143-154.

Ray, N.J., Jenkinson, N., Brittain, J., Holland, P., Joint, C., Nandi, D., Bain, P.G., Yousif, N. Green, A., Stein, J.S., Aziz, T.Z., 2009. The role of the subthalamic nucleus in response inhibition: evidence from deep brain stimulation for Parkinson's disease. Neuropsychologia 47, 2828-2834.

Rubia, K., Russell, T., Overmeyer, S., Brammer, M.J., Bullmore, E.T., Sharma, T., Simmons, A., Williams, S.C.R., Giampietro, V., Andrew, C.M., Taylor, E., 2001. Mapping motor inhibition: conjunctive brain activations across different versions of Go/No-Go and stop tasks. Neuroimage 13, 250-261.

Rubia, K., Smith, A.B., Brammer, M.J., Taylor, E., 2003. Right inferior prefrontal cortex mediates response inhibition while mesial prefrontal cortex is responsible for error detection. Neuroimage 20, 351-358.

Rubia, K., Smith, A.B., Taylor, E., Brammer, M., 2007. Linear age-correlated functional development of right inferior fronto-striato-cerebellar networks during response inhibition and anterior cingulate during error-related processes. Hum. Brain Mapp. 28, 1163-1177.

Schmajuk, M., Liotti, M., Busse, L., Woldorff, M.G., 2006. Electrophysiological activity underlying inhibitory control processes in normal adults. Neuropsychologia 44 384-395.

Stinear, C.M., Coxon, J.P., Byblow, W.D., 2009. Primary motor cortex and movement prevention: where Stop meets Go. Neurosci. Biobehav. Rev. 33, 662-673.

Verbruggen, F., Logan, G.D., 2008. Response inhibition in the stop-signal paradigm. Trends Cogn. Sci. 12, 418-424.

Verbruggen, F., Logan, G.D., 2009. Models of response inhibition in the stop-signal and stop-change paradigms. Neurosci. Biobehav. Rev. 33, 647-661.

Vink, M., Kahn, R.S., Raemaekers, M., van den Heuvel, M., Boersma, M., Ramsey, N.F. 2005. Function of striatum beyond inhibition and execution of motor responses. Hum. Brain Mapp. 25, 336-344.

Wager, T.D., Sylvester, C.Y., Lacey, S.C., Nee, D.E., Franklin, M., Jonides, J., 2005. Common and unique components of response inhibition revealed by fMRI. Neuroimage 27 323-340.

Williams, B.R., Ponesse, J.S., Schachar, R.J., Logan, G.D., Tannock, R., 1999. Development of inhibitory control across the life span. Dev. Psychol. 35, 205-213.

Xue, G., Aron, A.R., Poldrack, R.A., 2008. Common neural substrates for inhibition of spoken and manual responses. Cereb. Cortex 18, 1923-1932.

Yantis, S., 1993. Stimulus-driven attentional capture and attentional control settings. J. Exp. Psychol. Hum. Percept. Perform. 19, 676-681.

Yantis, S., Jonides, J., 1990. Abrupt visual onsets and selective attention: voluntary versus automatic allocation. J. Exp. Psychol. Hum. Percept. Perform. 16, 121-134.

Zheng, D., Oka, T., Bokura, H., Yamaguchi, S., 2008. The key locus of common response inhibition network for no-go and stop signals. J. Cogn. Neurosci. 20, 1434-1442. 